\title{
Simulating the All-Order Hopping Expansion II: Wilson Fermions
}

\author{
Ulli Wolff* \\ Institut für Physik, Humboldt Universität \\ Newtonstr. 15 \\ 12489 Berlin, Germany
}

\begin{abstract}
We investigate the extension of the Prokof'ev-Svistunov worm algorithm to Wilson lattice fermions in an external scalar field. We effectively simulate by Monte Carlo the graphs contributing to the hopping expansion of the twopoint function on a finite lattice to arbitrary order. Tests are conducted for a constant background field i. e. free fermions at some mass. For the method introduced here this is expected to be a representative case. Its advantage is that we know the exact answers and can thus make stringent tests on the numerics. The approach is formulated in both two and three space-time dimensions. In $D=2$ Wilson fermions enjoy special positivity properties and the simulation is similarly efficient as in the Ising model. In $D=3$ the method also works at sufficiently large mass, but there is a hard sign problem in the present formulation hindering us to take the continuum limit.
\end{abstract}

HU-EP-08/61

SFB/CCP-08-98

*e-mail: uwolff@physik.hu-berlin.de 


\section{Introduction}

To give a definition of quantum field theories beyond perturbation theory (Feynman diagrams) they normally have to be regularized by replacing space and usually also time by a lattice. Then the functional integral becomes a well-defined object and thus amenable to numerical methods, usually in the form of stochastically sampling lattice field configurations by Monte Carlo methods. Most theories of interest contain fermion fields which lead to some or all integration variables being anticommuting Grassmann 'numbers'. In the standard approach these integrations - possibly after introducing additional Bose fields - are Gaussian and are performed exactly. The result is an effective action of the bosonic fields alone which become coupled non-locally. The known Monte Carlo techniques to practically simulate such systems are mostly based on molecular dynamics and the hybrid Monte Carlo idea (HMC) [1], [2]. These methods have been improved and optimized rather successfully over the years by a very large effort of many members of the lattice community. On the other hand practitioners know that once fermions are decoupled then HMC for locally coupled Bose fields is not a very efficient algorithm compared to alternatives like over-relaxation which are then available, not to mention the (unfortunately few) cases where cluster methods can be applied. This implies a large penalty for fermions even in cases where their effects are only small. At small fermion masses the fermionic forces in HMC tend to grow and the step size of the molecular dynamics trajectories has to be taken small enough. With this quasi-continuous evolution one then has to be cautious about possible long autocorrelations. After all, to the best of the author's knowledge, the ergodicity of HMC has not been formally proven.

Maybe for the aforementioned reasons among others some part of the community has remained motivated to look for radically different approaches. A rather natural idea is to look for a representation of fermions as some sort of 'sum over configurations' more similar to the bosons. One of the pioneering papers developing such ideas is [3]. There as in numerous succeeding attempts one starts from an operator formulation of fermions and inserts intermediate states in the occupation number basis between factors of the transfer matrix. In this way occupied sites map out an ensemble of 'world-lines' or a gas of loops of fermions on the lattice. Often the amplitudes that arise oscillate in sign with the danger of leading to an unmanageable signal to noise ratio, the infamous fermionic sign problem. The inclusion of gauge fields in this approach poses additional problems.

A somewhat different approach was successful for - but also restricted to QCD at infinite gauge coupling, $\beta=0$, [4]. In the Euclidean path integral with staggered fermions but no gauge plaquette term the group valued gauge fields can be integrated out first. The resulting model of locally paired even Grassmann elements has contributions that can be viewed as a statistical system of explicitly 
color-neutral mesons (dimers) and baryon loops. Both these systems and the world-line gas à la [3] are difficult to simulate efficiently by local methods due to constraints which conflict with local deformations of the configurations. In some cases efficient nonlocal updates could be devised [5], [6].

A 'more Euclidean' version of the idea was proposed in [7]. These authors started from the determinant of the integrated-out staggered fermions and tried to stochastically generate its expansion into cycles. However the restriction to local updates and the sign problem, even for $D=2$ in this case, have limited the use of the method.

In [8] (see also [9]) a loop or world-line representation was proposed for the partition function of standard two-dimensional Euclidean Wilson fermions in an external scalar field. They were mapped on a certain 8-vertex model. Based on it the Gross-Neveu model was simulated with local updates in [10. In [11] the same representation was re-derived directly from the Grassmann integral for charge self-conjugate (Majorana) Wilson fermions. The mapping between Wilson fermions and a loop-gas could in addition be made precise also for a finite torus with (anti)periodic boundary conditions. A cluster algorithm for the loop-gas was developed in [11] which produces almost uncorrelated loop configurations at low cost. In the sequel Willi Rath and the author have tried to compute correlations based on these configurations [12]. The only solution we have found so-far proceeds via the numerical generation of the scalar $\sigma$-field that usually factorizes the GrossNeveu interaction. Then the close to singular Dirac operator in this random scalar field has to be inverted and the CPU time ends up being spent in a very similar fashion as in HMC.

In this paper, as an alternative approach, we adopt the 'worm' algorithm of Prokof'ev and Svistunov (PS) [13] to lattice fermions of the Wilson type. To this end we build on the study of the PS algorithm for the Ising model carried out as a preparation in [14]. While there the (untruncated) strong coupling expansion is sampled, the fermion loop-gas corresponds to the quite similar hopping expansion 1 . We here extend the loop-gas formulation of fermions on a torus in two ways. We generalize [11] to including two spinor field insertions at arbitrary lattice sites. It turns out that the PS algorithm is ideally suited to keep track of the non-local amplitudes involved due to Fermi statistics. The second non-trivial extension takes this construction to Majorana fermions in three Euclidean dimensions. While we understand why the fermionic sign problem mentioned before is absent in two dimensions if the system size is large in correlation lengths, the full problem has to be confronted in three dimensions. We indeed find for free fermions that are implemented numerically in this study, that the PS algorithm for $D=2$ is similarly efficient as in the Ising model. While clearly correct in principle also in

\footnotetext{
${ }^{1}$ Because of this strong similarity, we put this paper into one series with [14].
} 
$D=3$ it fails numerically with the present technique when the continuum limit is approached. We nonetheless find the three dimensional loop representation theoretically quite interesting. We think that the free Majorana fermion in $D=3$ is an excellent study ground for more clever techniques, for instance cluster improved observables, to still overcome the sign problem, perhaps along the lines of [15].

The organization of this paper is as follows. In the next section we set up our notation for the lattice fermions discussed followed by section 3 introducing dimers that label all possible hopping graphs needed for the PS simulation. Tools for the simulations are described in 4 . In section 5. we define the kind of observables on the loop ensemble that allow to make contact with fermionic two-point functions followed in 6 . by the description of numerical results. We end on 7 . conclusions including a brief outline how interaction can be added. In two appendices we collect the free fermion results used as benchmarks and a geometrical discussion of the fermionic phase factors arising for each closed loop.

\section{Majorana-Wilson lattice fermions}

We start from a standard Wilson-Dirac fermion with the action

$$
S_{\mathrm{WD}}=a^{D} \sum_{x} \bar{\psi}\left(\gamma_{\mu} \tilde{\partial}_{\mu}+m-\frac{r}{2} a \partial^{*} \partial\right) \psi
$$

We consider a $D$-dimensional standard hypercubic lattice with spacing $a$ in all directions and either periodic or antiperiodic boundary conditions for each direction over the respective periodicity length $L_{\mu}$. The boundary conditions are coded into a vector $\varepsilon_{\mu}$ with components 0,1 by the condition

$$
\psi\left(x \pm L_{\mu} \hat{\mu}\right)=(-1)^{\varepsilon_{\mu}} \psi(x)
$$

and similarly for $\bar{\psi}$, and $\hat{\mu}$ is a unit vector in the positive $\mu$ direction.

Unless stated otherwise, the mass $m$ is assumed to be a real $x$-dependent periodic external field $m(x)$ here. By later integrating over it with a suitable weight one can, starting from this building block, arrive at interacting theories like the Gross-Neveu model. The operators $\partial, \partial^{*}, \tilde{\partial}$ are the usual forward, backward, and symmetrized nearest neighbor differences. The set $\left\{\gamma_{\mu}, \mu=0,1, \ldots, D-1\right\}$ are hermitean Euclidean Dirac matrices. From here on we shall restrict ourselves to the space-time dimensions $D=2,3$ with $2 \times 2 \gamma$-matrices in both cases. The Wilson term suppresses the doublers and from here on we set its coefficient to the convenient value $r=1$.

The action (1) is invariant under charge conjugation for any $m(x)$. It is hence both possible and natural to split the fermion into two neutral Majorana compo- 
nents by setting

$$
\psi=\frac{1}{\sqrt{2}}\left(\xi_{1}+i \xi_{2}\right), \quad \bar{\psi}=\frac{1}{\sqrt{2}}\left(\xi_{1}^{\top}-i \xi_{2}^{\top}\right) \mathcal{C}
$$

with the charge conjugation matrix $\mathcal{C}$ obeying

$$
\mathcal{C} \gamma_{\mu} \mathcal{C}^{-1}=-\gamma_{\mu}^{\top}=-\gamma_{\mu}^{*}, \quad \mathcal{C}=-\mathcal{C}^{\top}
$$

Inserting this into (11) we find two identical contributions for $\xi_{1,2}$. In our Majorana reduction we consider only one such component in the following

$$
S=\frac{1}{2} a^{D} \sum_{x} \xi^{\top} \mathcal{C}\left(\gamma_{\mu} \tilde{\partial}_{\mu}+m-\frac{1}{2} a \partial^{*} \partial\right) \xi
$$

Note that the matrix in this quadratic form is antisymmetric. By collecting diagonal and neighbor terms we can rewrite this action as

$$
S=\frac{1}{2} \sum_{x}(D+m) \xi^{\top}(x) \mathcal{C} \xi(x)-\sum_{x, \mu} \xi^{\top}(x) \mathcal{C} P(\hat{\mu}) \xi(x+\hat{\mu})
$$

where we now have adopted lattice units $(a=1)$ and have introduced projectors

$$
P(n)=\frac{1}{2}\left(1-n_{\mu} \gamma_{\mu}\right) \quad\left(n^{2}=1\right)
$$

for each lattice direction $(n= \pm \hat{\mu})$. Note that the hopping term of a Majorana fermion is a function of the unoriented link because of the identity

$$
\xi^{\top}(x) \mathcal{C} P(\hat{\mu}) \xi(x+\hat{\mu})=\xi^{\top}(x+\hat{\mu}) \mathcal{C} P(-\hat{\mu}) \xi(x) .
$$

For $D=2$ the form (6) coincides with the starting point of [11].

To continue we introduce the shorthand notation

$$
\bar{\xi}=\xi^{\top} \mathcal{C}
$$

We emphasize that for the Majorana fermion this depends on $\xi$ while $\psi, \bar{\psi}$ were independent Grassmann integration variables. The partition function is given by

$$
Z_{0}^{(\varepsilon)}=\int D \xi \mathrm{e}^{-S}=\operatorname{Pf}\left[\mathcal{C}\left(\gamma_{\mu} \tilde{\partial}_{\mu}+m-\frac{1}{2} \partial^{*} \partial\right)\right]
$$

where the Gaussian integral over Majorana fields has led to a Pfaffian? of the antisymmetric matrix. The result depends on the boundary conditions, of course, which is exhibited for $Z_{0}^{(\varepsilon)}$ but left implicit on the right hand side.

\footnotetext{
${ }^{2}$ The order of factors in $D \xi$ is assumed to be such that this is true without an (irrelevant) extra sign.
} 
In a straight-forward generalization of [14] we now extend our study to include

$$
Z^{(\varepsilon)}(u, v)=\int D \xi \mathrm{e}^{-S} \xi(u) \bar{\xi}(v)
$$

which is a matrix in spin space. It is closely related to the two point function 3

$$
G(x, y ; m)=\langle\xi(x) \bar{\xi}(y)\rangle=\frac{Z^{(\varepsilon)}(x, y)}{Z_{0}^{(\varepsilon)}} .
$$

As we are considering bilinear fermions in an external field $m(x)$ the propagator can also be obtained as the solution of a system of linear equations

$$
\left(\gamma_{\mu} \tilde{\partial}_{\mu}+m-\frac{1}{2} \partial^{*} \partial\right) G(x, y ; m)=\delta_{x, y} \times 1_{\text {spin }}
$$

where the Dirac operator acts on $x$.

For constant $m$ such an evaluation can proceed by Fourier expansion and will serve us as a check below. Otherwise the Pfaffian is a problem similar to the fermion determinant and methods like HMC are suitable at least for an even number of flavors [16], [17]. Our objective here is however to develop a simulation method alternative to this approach.

We end this section with the remark that, in contrast to the Ising model, $Z^{(\varepsilon)}(x, x)$ is not equal to the ordinary partition function. Instead one may show that for any $m$

$$
Z^{(\varepsilon)}(x, x)=\frac{\partial Z_{0}^{(\varepsilon)}}{\partial m(x)} \quad \times 1_{\text {spin }}
$$

holds. To derive this relation we use that the space of antisymmetric $2 \times 2$ matrices is only one-dimensional, given by multiples of the second Pauli matrix. Hence in $Z^{(\varepsilon)}(x, x)$ the integral containing $\xi(x) \xi(x)^{\top}$ must be proportional to $\mathcal{C}^{-1}$.

\section{Dimer form of Majorana fermions}

We here derive the loop-gas form of the fermion correlation function and partition function. In principle this may be achieved by using theorems for the expansion of the Pfaffian together with the sparseness of the matrix introduced before. This would parallel the approach in [7]. Instead we shall extensively manipulate the representation by a Grassmann integral. This is physically more transparent and may be seen as deriving the required expansion formulas 'on the fly' as they are needed. The power of Grassmann numbers for such purposes was emphasized before in [18].

\footnotetext{
${ }^{3}$ The dependence of $G$ on the boundary conditions $\varepsilon$ is left implicit.
} 


\subsection{General structure}

We start from the factorized form

$$
Z^{(\varepsilon)}(u, v)=\int D \xi \prod_{z} \mathrm{e}^{-\frac{1}{2} \varphi(z) \bar{\xi}(z) \xi(z)}\left[\prod_{l=\langle x y\rangle} \mathrm{e}^{\bar{\xi}(x) P(\widehat{y-x}) \xi(y)}\right] \xi(u) \bar{\xi}(v)
$$

with the short hand

$$
\varphi(x)=D+m(x) .
$$

Because each $P$ is a one-dimensional projector and due to the Grassmann nature of $\xi$ there are only two terms 4 in the expansion of each link-factor. It can thus be 'dimerized'

$$
\mathrm{e}^{\bar{\xi}(x) P(\widehat{y-x}) \xi(y)}=\sum_{k_{l}=0,1}[\bar{\xi}(x) P(\widehat{y-x}) \xi(y)]^{k_{l}}
$$

leading to

$$
Z^{(\varepsilon)}(u, v)=\sum_{\left\{k_{l}\right\}} \int D \xi \prod_{z} \mathrm{e}^{-\frac{1}{2} \varphi \bar{\xi} \xi}\left[\prod_{l=\langle x y\rangle}[\bar{\xi}(x) P(\widehat{y-x}) \xi(y)]^{k_{l}}\right] \xi(u) \bar{\xi}(v) .
$$

For each configuration $\left\{k_{l}\right\}$ we say that links with $k_{l}=1$ carry an (active) dimer. Associated with each site we have only two Grassmann variables integrated over. This implies numerous constraints on contributing dimer configurations:

- at sites $x \notin\{u, v\}$ there can only be either 0 or 2 dimers adjacent

- if $u \neq v$, at these two sites there must be exactly 1 dimer touching

- at $u=v$ there can be no dimer touching.

In the simpler case of $Z_{0}^{(\varepsilon)}$ the analogous expansion requires 0 or 2 dimers around all sites. Any dimer configuration that obeys these conditions and contributes to $Z^{(\varepsilon)}(u, v)$ or to $Z_{0}^{(\varepsilon)}$ we call admissible.

As a consequence of these constraints dimers in admissible configurations have to form chains. These can never backtrack, intersect or overlap. For $u \neq v$ there must be exactly one chain or string connecting $u$ and $v$ that we call $\sigma$. Apart from it all other chains must form a number of closed loops $\lambda_{j}$. For contributions to $Z_{0}^{(\varepsilon)}$ there is no string but only loops and the same is true for $Z^{(\varepsilon)}(u, u)$ with the additional requirement that no loop passes through $u$.

It is to be emphasized that the string (if present) and the loops including their number is a unique one-to-one representation of an admissible dimer configuration,

$$
\left.\left\{k_{l}\right\}\right|_{\text {admissable }} \longleftrightarrow \sigma \cup\left\{\lambda_{j}, j=1,2, \ldots, N_{\lambda}\right\},
$$

where both the string and the set of loops can also be empty.

\footnotetext{
${ }^{4}$ While many of the previous steps go through also for $D=4$, we will need a third term here.
} 


\subsection{Amplitudes}

To evaluate a contribution for fixed admissible $\left\{k_{l}\right\}$, we can reorder freely all Grassmann bilinears like hopping terms of links with $k_{l}=1$, the local $\varphi \bar{\xi} \xi$ terms and the integration measure with two spin components $d \xi_{1} d \xi_{2}$ for each site. In this way the whole Grassmann integral is factorized into one site integrals that are carried out with the formula

$$
\int d \xi_{1} d \xi_{2} \xi \bar{\xi}=1_{\text {spin }}
$$

where we have chosen $\mathcal{C}_{12}=1=-\mathcal{C}_{21}$. At each monomer site - a site with no dimer adjacent - the integrations are saturated by a site-factor in (18) and contribute a factor $\varphi(z)=D+m(z)$.

Next we consider $\sigma$ and define $|\sigma|$ to be the number of dimers one has to cross to walk from $u$ to $v$. During the walk one encounters a sequence of sites $s_{i}$ separated by lattice unit vectors $n_{i}$,

$$
\text { string } \sigma \leftrightarrow\left\{u=s_{0}, s_{1}, s_{2}, \ldots, s_{|\sigma|}=v\right\}, \quad n_{i+1}=s_{i+1}-s_{i} .
$$

We notice that we may use (8) to our convenience along the path. Then, after carrying out the integrations belonging to all sites $s_{i}$, there emerges a product of projectors

$$
V(\sigma)=P\left(n_{1}\right) P\left(n_{2}\right) \cdots P\left(n_{|\sigma|}\right) .
$$

Each closed loop $\lambda_{j}$ can be labeled in exactly the same way except that now $s_{0}=s_{|\lambda|}$ holds (suppressing for the moment the loop index $j$ ). If we denote the sequence of unit shifts now by $\left\{m_{1}, m_{2}, \ldots, m_{|\lambda|}\right\}$, then closed loops contribute a scalar factor

$$
w\left(\lambda_{j}\right)=-\operatorname{tr}\left[P\left(m_{1}\right) P\left(m_{2}\right) \cdots P\left(m_{|\lambda|}\right)\right] .
$$

The minus sign here is the usual one coming from closed fermion loops. Technically speaking, upon closing the trace, one pair of $\xi, \bar{\xi}$ appears in the 'wrong' order. The cyclicity of the trace immediately implies, that $w\left(\lambda_{j}\right)$ is independent of where we start with $m_{1}$ along the loop. In addition one may use

$$
P(n)^{\top}=\mathcal{C} P(-n) \mathcal{C}^{-1}
$$

to show also independence of the direction chosen to traverse the loop. Hence $w\left(\lambda_{j}\right)$ is truly a function of the unoriented loop only.

\subsection{Evaluation of spin factors}

Using a bra-ket notation in spin space we write the Wilson projectors as

$$
P(m)=|m\rangle\langle m|, \quad\langle m \mid m\rangle=1, \quad m= \pm \hat{\mu}= \pm \hat{0}, \pm \hat{1}[, \pm \hat{2} \text { if } D=3] .
$$


Now the loop factor is composed of scalar products

$$
w\left(\lambda_{j}\right)=-\left\langle m_{1} \mid m_{2}\right\rangle\left\langle m_{2} \mid m_{3}\right\rangle \cdots\left\langle m_{|\lambda|-1} \mid m_{|\lambda|}\right\rangle\left\langle m_{|\lambda|} \mid m_{1}\right\rangle,
$$

i.e. factors associated with the sites connecting pairs of links met along the loop. The modulus of the individual factors is one where successive $m_{i}$ coincide (straight sections) and $1 / \sqrt{2}=\cos (\pi / 4)$ between orthogonal links (corners). This is easily seen from a simple example

$$
|\langle\hat{0} \mid \hat{1}\rangle|^{2}=\langle\hat{0}|P(\hat{1})| \hat{0}\rangle=\left\langle\hat{0}\left|\gamma_{0} P(\hat{1}) \gamma_{0}\right| \hat{0}\right\rangle=\langle\hat{0}|P(-\hat{1})| \hat{0}\rangle=\frac{1}{2}
$$

and similarly for any other orthogonal pair. With $\pi / 4$ we see the typical half-angle appearing with spinors. We thus find

$$
w\left(\lambda_{j}\right)=2^{-C\left(\lambda_{j}\right) / 2} \phi\left(\lambda_{j}\right),
$$

where $C\left(\lambda_{j}\right)$ is the number of corners around the loop and $\phi\left(\lambda_{j}\right)$ is a phase.

We now discuss a rather direct way to compute $\phi\left(\lambda_{j}\right)$. While it gives not much geometric insight into its meaning, this derivation will lend itself to a very direct algorithmic implementation. In appendix $B$ an alternative more geometrical analysis is presented.

We fix the ambiguous phases of $| \pm \hat{\mu}\rangle$ in a definite way, knowing that $w$ is independent of this convention. We start from $|\hat{0}\rangle$ with an arbitrary phase. Next one may demand a maximal number of five real positive phases

$$
\langle \pm \hat{1} \mid \hat{0}\rangle=\langle-\hat{0} \mid \hat{1}\rangle=\langle \pm \hat{2} \mid \hat{0}\rangle=\frac{1}{\sqrt{2}}
$$

This exhausts the free choices and the remaining phases, one in $D=2$ and additional six in $D=3$ can be evaluated. One possible way to do so is to construct all eigenvectors starting from $|\hat{0}\rangle$ with the help of the projectors:

$$
\begin{gathered}
| \pm \hat{1}\rangle=\sqrt{2} P( \pm \hat{1})|\hat{0}\rangle, \\
|-\hat{0}\rangle=\sqrt{2} P(-\hat{0})|\hat{1}\rangle=-\gamma_{1}|\hat{0}\rangle
\end{gathered}
$$

and

$$
| \pm \hat{2}\rangle=\sqrt{2} P( \pm \hat{2})|\hat{0}\rangle
$$

The implied phases can now be computed by just using the Dirac algebra and they are collected in table 1. In two dimensions only the upper left $4 \times 4$ block is relevant, where all phases are real, they belong to $\mathrm{Z}(2)$. In this case the sign for 


\begin{tabular}{|c|c|c|c|c|c|c|}
\hline & $|+\hat{0}\rangle$ & $|-\hat{0}\rangle$ & $|+\hat{1}\rangle$ & $|-\hat{1}\rangle$ & $|+\hat{2}\rangle$ & $|-\hat{2}\rangle$ \\
\hline$\langle+\hat{0}|$ & 1 & - & 1 & 1 & 1 & 1 \\
\hline$\langle-\hat{0}|$ & - & 1 & 1 & -1 & $i$ & $-i$ \\
\hline$\langle+\hat{1}|$ & 1 & 1 & 1 & - & $z$ & $z^{*}$ \\
\hline$\langle-\hat{1}|$ & 1 & -1 & - & 1 & $z^{*}$ & $z$ \\
\hline$\langle+\hat{2}|$ & 1 & $-i$ & $z^{*}$ & $z$ & 1 & - \\
\hline$\langle-\hat{2}|$ & 1 & $i$ & $z$ & $z^{*}$ & - & 1 \\
\hline
\end{tabular}

Table 1: Phases in all possible scalar products between eigenspinors.

each loop acquires a simple geometrical interpretation which will be discussed in section 5 .

In evaluating the phases involving the third dimension we have assumed that $\gamma_{1} \gamma_{2}=-i \gamma_{0}$ holds and set

$$
z=\frac{1+i}{\sqrt{2}}=\mathrm{e}^{i \frac{\pi}{4}}
$$

We cannot eliminate the complex phase factors by re-defining the phases of $| \pm \hat{2}\rangle$. There is another inequivalent irreducible Dirac representation in $D=3$ with the complex conjugate phases (for example from $\gamma_{\mu} \rightarrow-\gamma_{\mu}$ ). This means that the parity reflected loop has the opposite phase which is hence a pseudoscalar. The phase factors for 3-dimensional Wilson fermions are in $\mathrm{Z}(8)$ and all values are actually assumed for relatively short loops if non-planar ones are included. The group $\mathrm{Z}(8)$ is related to the rotation group being reduced to the lattice symmetries, see appendix B. Simple examples for loops with complex phases are shown in figure 1. They were extracted from a Monte Carlo simulation (see below) be 'tagging' the phase of configurations and plotting them.
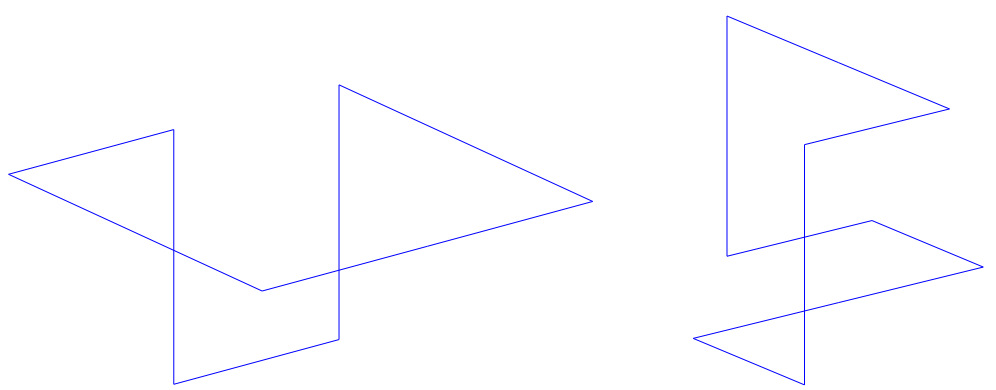

Figure 1: Closed fermion loops in $D=3$ with phase $\exp (i \pi / 4)$ (left) and $\exp (i \pi / 2)$ (right).

The spin factor for the open string from $u$ to $v$ is given by

$$
V(\sigma)=\left|n_{1}\right\rangle\left\langle n_{1} \mid n_{2}\right\rangle \cdots\left\langle n_{|\sigma|-1} \mid n_{|\sigma|}\right\rangle\left\langle n_{|\sigma|}\right| .
$$


For the leftmost ket and the rightmost bra we introduce the notation

$$
\left|n_{1}\right\rangle=|n(u)\rangle, \quad\left\langle n_{|\sigma|}\right|=\langle n(v)| \quad(u \neq v)
$$

such that $n(u)$ is the unit vector pointing out of $u$ in the direction of the unique adjacent dimer $k_{l}=1$ while $n(v)$ is the corresponding unit vector pointing toward $v$. Note that in principle we should write $n(u ; k)$ and $n(v ; k)$ and both vectors are undefined if $u=v$ holds. The scalar factors have again a modulus $2^{-1 / 2}$ for each corner and a phase $\phi(\sigma)$ that may be constructed from table 1 ,

$$
V(\sigma)=2^{-C(\sigma)} \phi(\sigma)|n(u)\rangle\langle n(v)| .
$$

We re-emphasize that all objects discussed above including the number of corners, the string and loop decomposition and the various phases are (nonlocal) functions of the $k_{l}$ in an admissible configuration. It would however clutter our notation too much to always exhibit this explicitly.

\subsection{Boundary conditions}

More phase factors can arise from boundary conditions if loops or the string winds around the torus in antiperiodic directions an odd number of times. We adopt the convention to label the points on the torus by coordinates $x_{\mu}=0,1, \ldots, L_{\mu}-1$ and distinguish a $(D-1)$-dimensional sheet of 'boundary' links for each direction as follows:

$$
l \text { is } a \text { boundary link in direction } \mu \leftrightarrow l=\langle x, x+\hat{\mu}\rangle \text { with } x_{\mu}=L_{\mu}-1 .
$$

For the string $\sigma$ and for each loop $\lambda_{j}$ we introduce parities $e_{\mu}(\sigma)$ and $e_{\mu}\left(\lambda_{j}\right)$ defined by

$$
e_{\mu}(\sigma)= \begin{cases}1 & \text { if } \sigma \text { contains an odd number of } \mu-\text { boundary links } \\ 0 & \text { else }\end{cases}
$$

and for the closed loops $e_{\mu}\left(\lambda_{j}\right)$ is completely analogous. The overall sign from the boundary conditions is now given by

$$
\operatorname{sign}=(-1)^{\varepsilon \cdot \bar{e}} \quad \text { with } \quad \bar{e}_{\mu}=e_{\mu}(\sigma)+\sum_{j=1}^{N_{\lambda}} e_{\mu}\left(\lambda_{j}\right) \quad(\bmod 2)
$$

with the scalar product of the $D$-vectors $\varepsilon_{\mu}$ and $\bar{e}_{\mu}$ in the exponent.

All contributions to the amplitude of an admissible dimer configuration have now been identified and will be combined in the next section to represent fermionic quantities in an ensemble summing and ultimately sampling such configurations.

\footnotetext{
${ }^{5}$ Of course, the torus has no boundary, hence the quotes.
} 


\section{Dimer partition function and worm algorithm}

\subsection{Dimer partition function}

Next we formally write down the characteristic function $\Theta(k ; u, v)$ that is unity for admissible configurations and zero for all others. In principle it has been defined before in words. As a building block we use

$$
d(k ; x)=\sum_{l, \partial l \ni x} k_{l}
$$

which counts the number of dimers adjacent at $x$. Then we define

$$
\begin{aligned}
& \Theta(k ; u, v)=\delta_{d(k ; u), 1} \delta_{d(k ; v), 1} \prod_{x \notin\{u, v\}}\left(\delta_{d(k ; x), 0}+\delta_{d(k ; x), 2}\right) \quad \text { for } u \neq v \\
& \Theta(k ; u, v)=\prod_{x}\left(\delta_{d(k ; x), 0}+\delta_{d(k ; x), 2}\right) \quad \text { for } u=v .
\end{aligned}
$$

Note that the constraint enforced for $u=v$ here is the one for contributions to $Z_{0}^{(\varepsilon)}$ rather than the more restrictive one for contributions to $Z^{(\varepsilon)}(u, u)$. We now consider the following partition function

$$
\mathcal{Z}=\sum_{u, v,\left\{k_{l}\right\}} \frac{\Theta(k ; u, v)}{\rho(u, v)} 2^{-\bar{C} / 2} \prod_{x, d(k ; x)=0} \varphi(x) .
$$

Here $\bar{C}$ is the total number of corners

$$
\bar{C}=C(\sigma)+\sum_{j=1}^{N_{\lambda}} C\left(\lambda_{j}\right)
$$

$\rho$ is an arbitrary symmetric strictly positive lattice-periodic function similar as in [14. The product is the weight from all monomer sites and we here restrict ourselves to

$$
\varphi(x)=D-m(x)>0
$$

guaranteeing the positivity of the overall weight. Expectation values of observables $A(k ; u, v)$ in this ensemble are defined by

$$
\langle\langle A\rangle\rangle=\frac{1}{\mathcal{Z}} \sum_{u, v,\left\{k_{l}\right\}} A(k ; u, v) \frac{\Theta(k ; u, v)}{\rho(u, v)} 2^{-\bar{C} / 2} \prod_{x, d(k ; x)=0} \varphi(x) .
$$

Observables related to the Majorana fermions discussed before can be written as ratios of such expectation values. This will be discussed in the next section after introducing the simulation algorithm for (43). 


\subsection{Prokof'ev-Svistunov worm algorithm}

The simulation of the dimer ensemble can be carried out with the worm algorithm of PS [13]. It is very similar to the algorithm described in [14] and we can be brief here about details. The main difference to the Ising case is that more than 2 dimers cannot touch and that there is a weight $1 / \sqrt{2}$ for corners which induces a kind of stiffness (tendency to be straight) of the chains.

We briefly pause here to comment on the notation of hopping parameter expansion in the title of the paper. The factors $\varphi(x)$ appearing for monomers could be rescaled to unity by absorbing them into $\xi(x)$ early on. Then each dimer $k_{\langle x y\rangle}$ would be accompanied by a factor $[\varphi(x) \varphi(y)]^{-1 / 2}$. For constant $m$ this would equal $[D+m]^{-1}=2 \kappa$ with the conventional hopping parameter $\kappa$. Thus $2 \kappa$ is the strict analog of the Ising strong coupling expansion parameter $\tan \beta$ in [14]. We prefer however to stay with the unrescaled form which is advantageous for the introduction of interaction via $m(x)$.

An update microstep of the PS algorithm is now a succession of steps I and II applied to admissible configurations. In step I we make a Metropolis decision on a proposal where we pick one of the $2 D$ nearest neighbors of $v$ with equal probability and call it $v^{\prime}$ and the connecting link $l$. The proposed move changes $v \rightarrow v^{\prime}$ flipping at the same time $k_{l} \rightarrow k_{l}^{\prime}=1-k_{l}$. It brings us from the global configuration $k$ to configuration $k^{\prime}$ (differing at exactly one link). Note that $k^{\prime}$ may be not admissible, in which case the move will be rejected. We first form an auxiliary quantity $q$, the ratio of amplitudes after and before the move. We have to distinguish a number of cases and collect values of $q$ in table 2, We have written $n\left(u^{\prime}\right)$ as a shorthand for what should be $n\left(u ; k^{\prime}\right)$ after the move, although we did not move $u$ here. The allowed moves are illustrated in figure 2 . Parts a), b), c) refer to lines 1, 3, 5 of the table. The lines below those refer to the reverse changes. They correspond to the same graphs read from right to left with the arrow reversed and interchanged $v \leftrightarrow v^{\prime}$. The directions of active dimers not participating in the present update are examples only and can also point differently.

In all other cases not covered here $q$ is set to zero. In lines 3 and 4 of the table a sign is included for changing the number of fermion loops $N_{\lambda}$ by one. Finally the modulus of $q$ is used in the acceptance probability

$$
p_{\text {acc }}=\min \left(1, \frac{\rho(u, v)}{\rho\left(u, v^{\prime}\right)}|q|\right)
$$

while the phase changes will be considered in the next section.

The type II move is as follows. If we encounter a configuration $u, v,\left\{k_{l}\right\}$ with $u=v$ we 'kick' $u=v$ together to a randomly chosen other lattice site with unchanged $\left\{k_{l}\right\}$ with the probability $0<p_{\text {kick }} \leqslant 1$. For $u \neq v$ we do nothing in this step, which is the dominant case. A difference with respect to the Ising case 


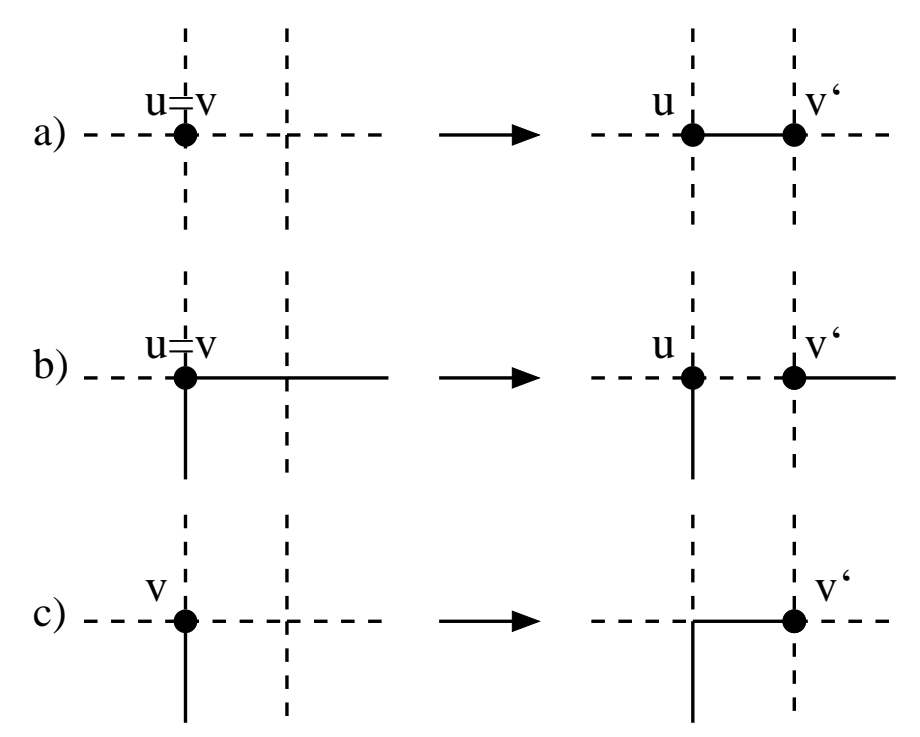

Figure 2: Pictorial representation of elementary moves in the PS algorithm. Solid lines are active dimers (value one).

is that while there also $p_{\text {kick }}=0$ (absence of step II) yields an ergodic algorithm [19], this is not so here. For the fermions the jumps are required to move between different connected components.

Moving only $v$ together with steps II constitutes a correct Monte Carlo algorithm. We nevertheless found it advantageous to also move $u$ in a completely analogous fashion. We thus now call the sequence $\mathrm{I}_{u}-\mathrm{II}-\mathrm{I}_{v}-$ II a microstep and call $N_{x} / 2$ mircosteps an iteration if we have $N_{x}$ lattice sites.

We found the choice of $p_{\text {kick }} \in[0.3,1]$ not critical and use $p_{\text {kick }}=0.7$ in the following after a few quick experiments.

\begin{tabular}{|c|c|c|c|c|}
\hline$d(k ; v)$ & $d\left(k ; v^{\prime}\right)$ & $d\left(k^{\prime}, v\right)$ & $d\left(k^{\prime} ; v^{\prime}\right)$ & $q$ \\
\hline 0 & 0 & 1 & 1 & {$\left[\varphi(v) \varphi\left(v^{\prime}\right)\right]^{-1}$} \\
\hline 1 & 1 & 0 & 0 & $\varphi(v) \varphi\left(v^{\prime}\right)$ \\
\hline 2 & 2 & 1 & 1 & $-\left[\left\langle n\left(v^{\prime}\right) \mid v-v^{\prime}\right\rangle\left\langle v-v^{\prime} \mid n\left(u^{\prime}\right)\right\rangle\right]^{-1}$ \\
\hline 1 & 1 & 2 & 2 & $-\left\langle n(v) \mid v^{\prime}-v\right\rangle\left\langle v^{\prime}-v \mid n(u)\right\rangle$ \\
\hline 1 & 0 & 2 & 1 & $\left\langle n(v) \mid v^{\prime}-v\right\rangle\left[\varphi\left(v^{\prime}\right)\right]^{-1}$ \\
\hline 1 & 2 & 0 & 1 & $\varphi(v)\left[\left\langle n\left(v^{\prime}\right) \mid v-v^{\prime}\right\rangle\right]^{-1}$ \\
\hline
\end{tabular}

Table 2: Entries in the first four columns specify the condition for possible moves, under which the amplitude gets multiplied by $q$ (not including possible signs from antiperiodic boundary conditions). 


\section{$5 \quad$ Fermionic phase and spin factors}

\subsection{Formulae for both $D=2$ and $D=3$}

The configurations of the dimer ensemble just discussed correspond to the set of graphs of the hopping parameter expansion of Majorana fermions. Each admissible configuration contributes to $Z_{0}^{(\varepsilon)}$ or to $Z^{(\varepsilon)}(u, v)$ with a certain amplitude and in the second case also with a spin matrix. The moduli of the amplitudes have been incorporated into the generation of configurations. The phases of the amplitudes and the spin matrices will be taken into account now as observables evaluated as in (46).

We first combine all phases discussed in section 3.3 and 3.4 into the total phase

$$
\Phi^{(\varepsilon)}(k)=(-1)^{\varepsilon \cdot \bar{e}} \phi(\sigma) \prod_{j=1}^{N_{\lambda}} \phi\left(\lambda_{j}\right) .
$$

For $u=v$ we set $\phi(\sigma)=1$, there is no string, only loops.

As mentioned before for $D=2$ the phase $\Phi$ is just a sign while for $D=3$ it is an element of $\mathrm{Z}(8)$. We now have the connection

$$
V Z_{0}^{(\varepsilon)}=\mathcal{Z}\left\langle\left\langle\rho(u, u) \delta_{u, v} \Phi^{(\varepsilon)}(k)\right\rangle\right\rangle
$$

with the volume

$$
V=\prod_{\mu=0}^{D-1} L_{\mu}
$$

If we define a spin matrix

$$
\mathcal{S}(k ; u, v)=\left\{\begin{array}{lll}
|n(u)\rangle\langle n(v)| & \text { if } \quad u \neq v \\
\delta_{d(k ; u), 0}[\varphi(u)]^{-1} 1_{\text {spin }} & \text { if } \quad u=v
\end{array}\right.
$$

the cases with insertions may be uniformly written as

$$
Z^{(\varepsilon)}(x, y)=\rho(x, y) \mathcal{Z}\left\langle\left\langle\delta_{u, x} \delta_{v, y} \Phi^{(\varepsilon)}(k) \mathcal{S}(k ; u, v)\right\rangle\right\rangle .
$$

At coinciding arguments $x=y$ the Grassmann integrations are saturated by the insertion alone, which requires a monomer site with its usual weight factor to be canceled. In this case one could in principle also relax the constraint in (52) to obtain

$$
V Z^{(\varepsilon)}(x, x)=\mathcal{Z}\left\langle\left\langle\rho(u, u) \delta_{u, v} \Phi^{(\varepsilon)}(k) \mathcal{S}(k ; x, x)\right\rangle\right\rangle .
$$

For the fermion correlation function the connection is

$$
G(x, y ; m)=\frac{\rho(x, y)\left\langle\left\langle\delta_{u, x} \delta_{v, y} \Phi^{(\varepsilon)}(k) \mathcal{S}(k ; u, v)\right\rangle\right\rangle}{(1 / V)\left\langle\left\langle\rho(u, u) \delta_{u, v} \Phi^{(\varepsilon)}(k)\right\rangle\right\rangle} .
$$


An alternative derivation of the result for coinciding points starts from the observation

$$
-\frac{1}{2}\langle\bar{\xi} \xi(x)\rangle=\frac{\partial}{\partial m(x)} \ln Z_{0}^{(\varepsilon)}=\frac{1}{\varphi(x)} \frac{\left\langle\left\langle\rho(u, u) \delta_{u, v} \Phi^{(\varepsilon)}(k) \delta_{d(k ; x), 0}\right\rangle\right\rangle}{\left\langle\left\langle\rho(u, u) \delta_{u, v} \Phi^{(\varepsilon)}(k)\right\rangle\right\rangle}
$$

and uses (49) and (14).

From the structure of the contributions in the dimer ensemble we may conclude that the right hand side of (54) is rational in the external field $m(x)$. The denominator has total degree $V$, the numerator $V-1-|\sigma|_{\min }$. Here $|\sigma|_{\min }$ is the minimal number of links to connect $x$ and $y$ by a string. The degree in each individual $m(x)$ is only linear both in the numerator and denominator.

The above formulae simplify if translation invariance holds, $m(x) \equiv m, G(x, y ; m)$ $\rightarrow G(x-y)$, where we also restrict $\rho(x, y)=\rho(x-y)$ and normalize $\rho(0)=1$. We then find

$$
G(z)=\rho(z) \frac{\left\langle\left\langle\delta_{u-v, z}^{(\varepsilon)} \Phi^{(\varepsilon)}(k) \mathcal{S}(k ; u, v)\right\rangle\right\rangle}{\left\langle\left\langle\delta_{u, v} \Phi^{(\varepsilon)}(k)\right\rangle\right\rangle} .
$$

We recognize close similarities with the Ising correlation in 14 with the novelty of averaging the phasefactor and the spin matrices. Note that the delta function $\delta^{(\varepsilon)}$ in the numerator needs to have the same antiperiodicity as the fields $\xi$.

From (49) one may now trivially read off that

$$
\frac{Z_{0}^{(\varepsilon)}}{Z_{0}^{\left(\varepsilon^{\prime}\right)}}=\frac{\left\langle\left\langle\delta_{u, v} \Phi^{(\varepsilon)}(k)\right\rangle\right\rangle}{\left\langle\left\langle\delta_{u, v} \Phi^{\left(\varepsilon^{\prime}\right)}(k)\right\rangle\right\rangle}
$$

allows to measure the change in free energy for different boundary conditions.

This type of quantity is theoretically nice, because it is expected to possess a continuum limit in a finite volume. For the massless all-periodic case $\varepsilon_{\mu} \equiv 0$, the partition function $Z_{0}^{(\varepsilon)}$ vanishes at $m=0$ because the matrix under the Pfaffian then has two exact zero modes. The corresponding phasefactor then averages to zero exactly.

\section{$5.2 \quad D=2$ specialties}

Fermions in two Euclidean or one space dimension are simpler and in a way untypical for the true problem related to the oscillating phase $\Phi$. In the Euclidean field theory formulation this is seen by the phases from fermion loops and from spin 'essentially canceling' in $D=2$ (only). In our realization this is seen as follows. Minus signs appear only at two types of corners, namely $\langle-\hat{0} \mid-\hat{1}\rangle$ or $\langle-\hat{1} \mid-\hat{0}\rangle$. By drawing closed loops with the intersection properties relevant here on a planar torus it is not difficult so see that 
- loops winding around the torus in one or both dimensions receive an even number of such minus signs

- loops that close trivially and do not wind around the torus receive an odd number of minus signs from spin phases.

In [11] a more detailed discussion of this and some illuminating figures with examples can be found. Winding around the torus can be read off from the crossing of 'boundary links' (38). Thus the result for each closed loop on the two-dimensional torus can be summarized in our notation as

$$
\phi\left(\lambda_{j}\right)=\left\{\begin{array}{ll}
+1 & \text { if } \\
-1 & \text { else }
\end{array} e_{\mu}\left(\lambda_{j}\right)=(0,0) \quad(D=2 \text { only }) .\right.
$$

Negative signs - remember that the Fermi loop sign has been included in $\phi-$ only come from topologically nontrivial loops that cannot be contracted to the trivial loop by series of plaquette moves [10], [11]. The total phase for $u=v$ configurations can now be given as

$$
\Phi^{(\varepsilon)}(k)=(-1)^{\varepsilon \cdot \bar{e}+\delta_{\bar{e},(0,0)}+1} \quad[\text { for } u=v, \Theta(k ; u, u)=1] .
$$

It depends on $k$ only via the topology variable $\bar{e}_{\mu}$. Using Fourier transformation on $\mathrm{Z}(2)\left[\sum_{\varepsilon}(-1)^{\varepsilon \cdot\left(e-e^{\prime}\right)}=4 \delta_{e, e^{\prime}}\right]$ one may show the identity

$$
1 \equiv \sum_{\varepsilon} z(\varepsilon) \Phi^{(\varepsilon)}(k), \quad z(\varepsilon)=\frac{1}{2}(-1)^{\delta_{\varepsilon,(0,0)}}
$$

for this case. This in turn implies for the average monomer density (with no phases)

$$
\bar{K}=\frac{1}{V} \frac{\left\langle\left\langle\delta_{u, v} \sum_{x} \delta_{d(k ; x), 0}\right\rangle\right\rangle}{\left\langle\left\langle\delta_{u, v}\right\rangle\right\rangle}
$$

the exact result (for free fermions)

$$
\bar{K}=\frac{2+m}{V} \frac{\partial}{\partial m} \ln \bar{Z}_{0}
$$

with the partition function

$$
\bar{Z}_{0}=\sum_{\varepsilon} z(\varepsilon) Z_{0}^{(\varepsilon)}
$$

summed over boundary conditions with amplitudes $z(\varepsilon)$. Similarly from (57) we may deduce now

$$
\frac{Z_{0}^{(\varepsilon)}}{\bar{Z}_{0}}=\frac{\left\langle\left\langle\delta_{u, v} \Phi^{(\varepsilon)}(k)\right\rangle\right\rangle}{\left\langle\left\langle\delta_{u, v}\right\rangle\right\rangle} .
$$




\section{$6 \quad$ Numerical experiments}

In this section we test our simulation method for the case of free fermions on various lattice sizes and (constant) $m$ values in both $D=2$ and 3 . While of course no numerical simulations are really needed here, we nonetheless think that for our loop-gas representation this is not an untypical case also for later interacting applications. Thus the advance knowledge of the results here is just an advantage for precision testing.

To extract fermionic quantities from simulations we must have the phase $\Phi^{(\varepsilon)}(k)$ available for each sampled configuration for the desired boundary conditions. For each admissible configuration with $\Theta(k ; u, v)=1$ it can be constructed by tracing the string and all loops at the cost of order $V$ operations. It is however easier to update its value together with the configurations. In fact this is even necessary to measure efficiently between microsteps as discussed in [14]. To that end we assume $\Phi^{(\varepsilon)}(k)$ to be known for the start configuration. We always took the trivial $u=v=0, k_{l} \equiv 0$ with $\Phi^{(\varepsilon)}(k)=1$. Then, whenever an update proposal of type $\mathrm{I}_{v}$ of the PS algorithm is accepted, we change

$$
\Phi^{(\varepsilon)}(k) \rightarrow \Phi^{(\varepsilon)}\left(k^{\prime}\right)=\Phi^{(\varepsilon)}(k) \times \frac{q}{|q|} \times \eta\left(\left\langle v v^{\prime}\right\rangle, \varepsilon\right)
$$

and similarly for $\mathrm{I}_{u}$. Here $q$ is given in table 2 and the additional factor

$$
\eta\left(\left\langle v v^{\prime}\right\rangle, \varepsilon\right)=\left\{\begin{array}{ll}
-1 & \text { if } \\
+1 & \text { else }
\end{array} \quad\left\langle v v^{\prime}\right\rangle \text { is a } \mu-\text { boundary link and } \varepsilon_{\mu}=1\right.
$$

takes into account the boundary conditions (see (38)). Needless to say, one may also keep track of $\Phi^{(\varepsilon)}$ for several boundary conditions in the same run, as the updates do not depend on them.

The spin matrix is easy to construct at any time from the single dimers adjacent to $u$ and $v \neq u$ and is trivial for the coinciding case. In practice we measure correlations contracted with some Dirac matrix $\Gamma$ which leads to

$$
-\langle\bar{\xi}(0) \Gamma \xi(z)\rangle=\rho(z) \frac{\left\langle\left\langle\delta_{u-v, z}^{(\varepsilon)} \Phi^{(\varepsilon)}(k) \operatorname{tr}[\mathcal{S}(k ; u, v) \Gamma]\right\rangle\right\rangle}{\left\langle\left\langle\delta_{u, v} \Phi^{(\varepsilon)}(k)\right\rangle\right\rangle} .
$$

We want to further specialize to zero spatial momentum as discussed in appendix A.

$$
k_{\Gamma}\left(z_{0}\right)=-\sum_{z_{k}}\langle\bar{\xi}(0) \Gamma \xi(z)\rangle \propto \rho\left(z_{0}\right)\left\langle\left\langle\delta_{u_{0}-v_{0}, z_{0}}^{(\varepsilon)} \Phi^{(\varepsilon)}(k) \operatorname{tr}[\mathcal{S}(k ; u, v) \Gamma]\right\rangle\right\rangle .
$$

We took $\rho$ to only depend on time and dropped the denominator. For symmetry reasons only $\Gamma=1, \gamma_{0}$ (also labeled as $S, V$ ) leading to the scalar and vector correlations $k_{S}, k_{V}$ are nontrivial. During the simulation we simply add the corresponding 
amplitudes into bins for each separation $z_{0}$ and then end up with correlations measured for all distances. Pre-computed tables are heavily used to speed up and they lead to a very simple code. In the simulations reported below we have observed Metropolis acceptance rates close to $50 \%$ for $D=2$ and $30 \%$ for $D=3$. This is close to the amplitude change by $1 /(D+m)$ when the worm 'eats' a monomer which it has to do to grow. In equilibrium also the other processes are important, but this one seems to set the scale.

All error estimates below are derived with the method and tools detailed in [20]. In particular the definition of integrated autocorrelation times $\tau_{\text {int }}$ employed here can be found, see also remarks in [14. Due to time series of length $10^{6}$ and more the convolution step in UWerr, eq. (31) in [20], became a bit slow for online data analysis. We therefore tailored a special version UWerr_fft which accelerates this step by using the fast Fourier transform. It is available on the web under www .physik.hu-berlin.de/com/ALPHAsoft.
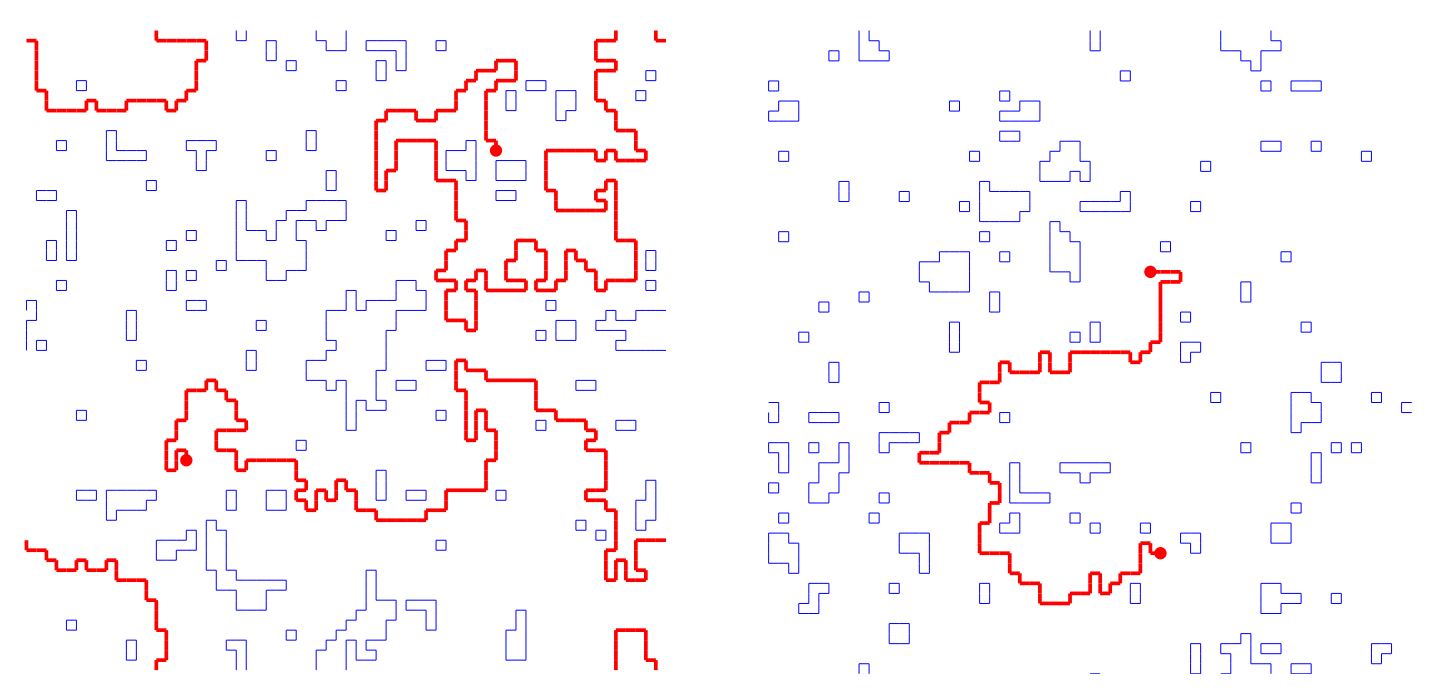

Figure 3: Two typical configurations on a $64^{2}$ lattice at criticality $(m=0$, left panel) and with correlation length $64 / 5(m=0.0812 \ldots$, right panel $)$. The string $\sigma$ is given by the fat (red line), the other lines are loops $\lambda_{j}$. Readers are asked to identify left-right and top-bottom edges in their mind.

\section{1 $D=2$, physically large volume}

For tests in this subsection we chose a mass such that $\omega L=5$ holds with the pole mass $\omega=\ln (1+m)$. The zero momentum timeslice correlations (68) then fall off exactly with $\exp \left(-\omega x_{0}\right)$, modified to cosh or sinh due to time periodicity, see appendix A. A typical configuration is visualized by the right picture in figure 
3. We see that for fermions, in contrast to the Ising model, there really is a unique 'worm', which moves by the updates. In all simulations the complete zero momentum two-point functions at all separations were consistent within errors with the exact results. We routinely checked plots of the deviation in units of the estimated error against $x_{0}$ which are order one, occasionally straddling \pm 2 . In addition diagnostic quantities like (61) and (64) were monitored. Because in a large box (compared to the inverse mass) few configurations wind around the torus we find no significant difference between periodic and antiperiodic boundary conditions. In the example to follow we measured (64) and obtained

$$
\frac{Z_{0}^{(0,0)}}{\bar{Z}_{0}}=0.9744(5), \quad \frac{Z_{0}^{(1,0)}}{\bar{Z}_{0}}=0.9745(5)
$$

in agreement with the exact answer.
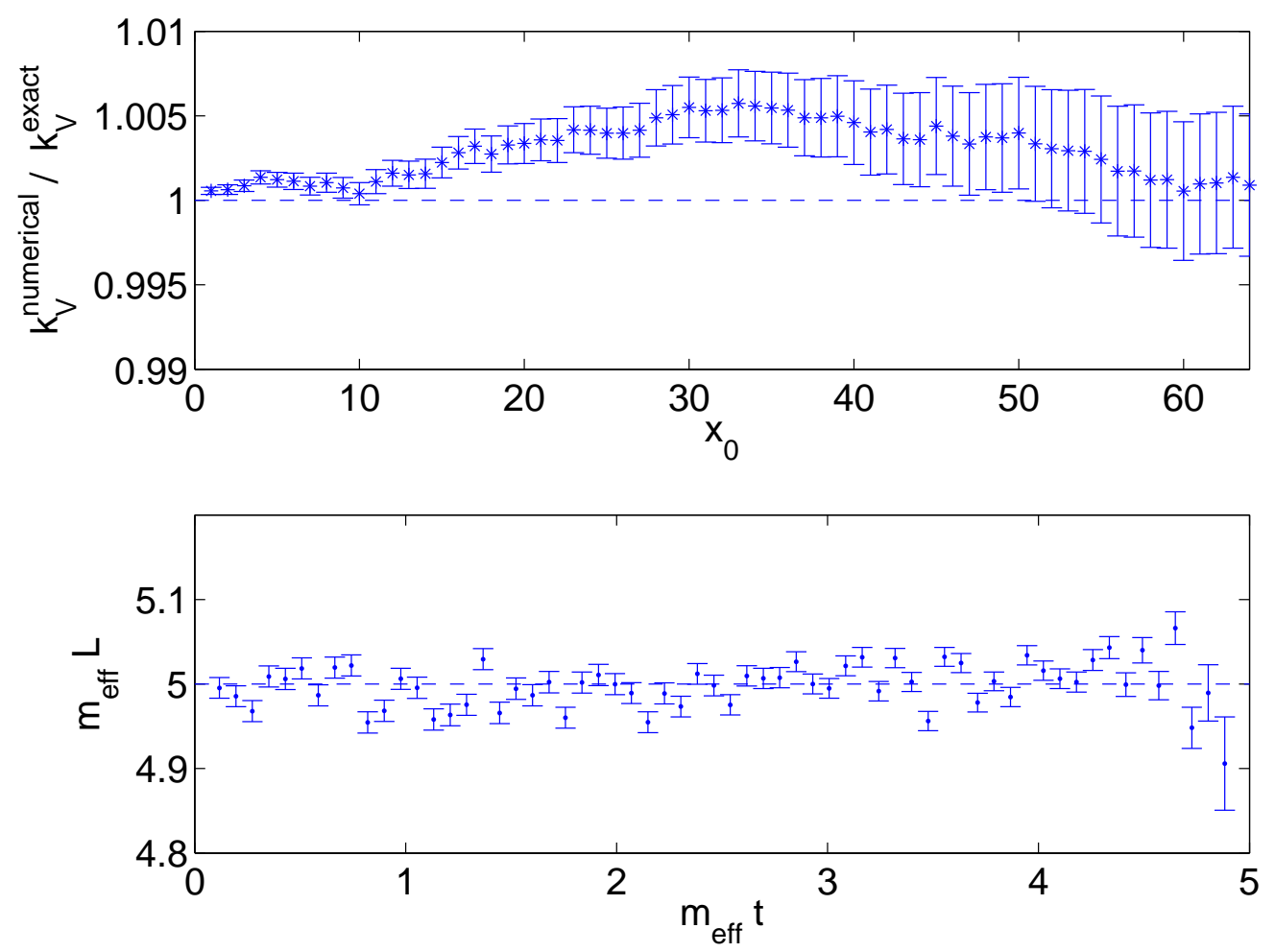

Figure 4: Correlation function $k_{V}$ and the effective mass derived from it. Errorbars are one sigma high.

As an impression for the reader we show in figure 4 results for the vector correlation $k_{V}$ on a lattice $L=64, T=2 L$ with $\varepsilon_{\mu}=(1,0)$ after accumulating $10^{7}$

\footnotetext{
${ }^{6}$ The poor unoriented Majorana worm has however no distinction between head and tail!
} 
iterations (steps per site). The correlation length $\omega^{-1}$ is hence about 13 lattice spacings. We have chosen the bias $\rho$ as

$$
\rho(t) \propto \cosh [\omega(T / 2-t)]
$$

which leads to a population of timeslices $\left\langle\left\langle\delta_{u_{0}-v_{0}, t}\right\rangle\right\rangle$ approximately flat in $t$. We refer the reader to the discussion in [14], which can be taken over essentially without change. The upper panel shows the correlation $k_{V}\left(x_{0}\right)$ itself normalized by its exact value. The growth of the errors from left to right is due to an increase of the integrated correlation time $\tau_{\text {int }}$ from about 0.6 iterations at short distances to about 15 iterations at $x_{0}=T / 2$. In the lower panel we give the effective mass as a function of distance by matching subsequent timeslices to

$$
\frac{k_{V}\left(x_{0}+1\right)}{k_{V}\left(x_{0}\right)}=\frac{\cosh \left(m_{\mathrm{eff}}\left(T / 2-x_{0}-1\right)\right.}{\cosh \left(m_{\mathrm{eff}}\left(T / 2-x_{0}\right)\right.}, \quad 0<m_{\mathrm{eff}} \equiv m_{\mathrm{eff}}\left(x_{0}+1 / 2\right) .
$$

Here errors appear (apart from $x_{0}$ very close to $T / 2$ ) to be independent of the separation in agreement with the observed autocorrelations $\tau_{\text {int }} \approx 0.5$ for all $x_{0}$. The longer autocorrelations observed in $k_{V}$ apparently cancel in the ratio. From the fluctuations in figure 4 we conclude qualitatively that statistical fluctuations at neighboring time separations are strongly correlated in $k_{V}$, but much less so in $m_{\text {eff }}$. In a run with $\rho \equiv 1$ the growth of $\tau_{\text {int }}$ for $k_{V}$ does not occur. Its error however grows in a similar way due to the larger variance coming from fewer data at large separation $u-v$ (fewer 'long worms') when no bias $\rho$ is applied. The more interesting effective mass is more accurate with the bias used for the figure.

\section{2 $D=2$, physically small volume}

We now simulate at the critical point which in the free case is known to occur at $m=0$. We remind that due to the infrared regulator given by the small inverse temperature $T$ with antiperiodic boundary conditions $\varepsilon_{0}=1$ this is physically well-defined. Such applications are of interest in interacting theories to study nonperturbative renormalization using the universal finite volume continuum limit. To this end we report measurements of $k_{S}(T / 4)$ and $k_{V}(T / 2)$ with $\varepsilon=(1,0)$. Further motivation for the study of these objects can be found in appendix $\mathrm{A}$ and refs. [16], [17]. In table 3 we compile our results from performing $10^{6}$ iterations at each of the lattice sizes. Again $\tau_{\text {int }}$ are given in iterations.

Here the topology and the sign $\Phi^{(1,0)}$ fluctuate, but we can achieve a percent accuracy with the given statistics, which could be enlarged. 


\begin{tabular}{|c|c|c|c|c|}
\hline$T=L$ & $k_{S}(T / 4)$ & $\tau_{\text {int }, k_{S}(T / 4)}$ & $k_{V}(T / 2)$ & $\tau_{\text {int }, k_{V}(T / 4)}$ \\
\hline 16 & $0.010(7)$ & $1.85(8)$ & $0.997(10)$ & $1.11(2)$ \\
\hline 32 & $-0.011(15)$ & $3.6(4)$ & $1.007(16)$ & $1.43(4)$ \\
\hline 64 & $0.011(25)$ & $5.0(5)$ & $0.943(29)$ & $2.68(14)$ \\
\hline
\end{tabular}

Table 3: Results at the critical point $m=0$. The exact values for all $L$ are $k_{S}(T / 4)=0$ due to chiral symmetry and $k_{V}(T / 2)=1$ corresponding to canonical field normalization.

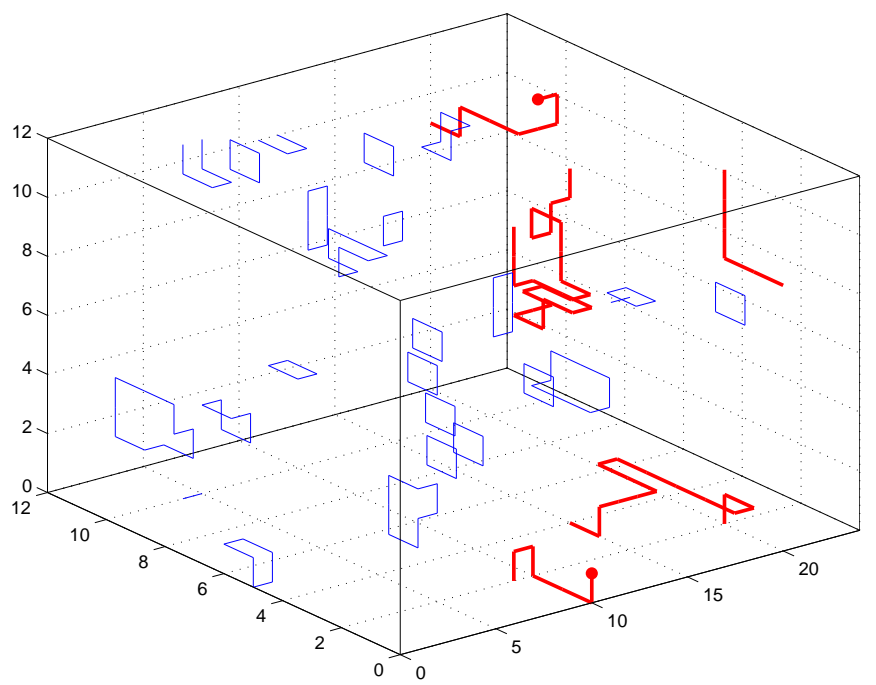

Figure 5: A typical configuration on $24 \times 12^{2}$ with correlation length 2 ( $\mathrm{m}=$ $0.6487 \ldots)$.

\section{3 $D=3$, sign problem}

It is trivial to adapt the code from two to three dimensions. On small lattices $T, L=4,6$ we performed similar validation tests as before with completely accurate and satisfactory results. Note that the formulae for $k_{S}, k_{V}$ in appendix $\mathrm{A}$ are equally valid for $D=2,3$. It turns out, however, that now very suddenly as the volume is increased or the mass is lowered the sign fluctuations abruptly become so violent that no signal is left in (67) and also for ratios of correlations as in (70) all estimates yield ' $0 / 0$ ' within errors: the sign problem. For large enough mass loops remain small and predominantly planar. Such loops are as in two dimensions with phase one. For a demonstration we show in table 4 results for two cases just before trouble strikes. In figure 5 the last configuration of our run at $\omega L=6$ is shown. For $\omega L=4$ and the same lattice size no meaningful results can be obtained anymore. Integrated autocorrelations times were close to $1 / 2$ for all the quantities studied. Although the observables in the dimer ensemble are complex, 
the averages of the imaginary parts vanish within errors as they have to, since they are parity odd. This was first checked and then used before forming quotients. A bias was not used here, $\rho \equiv 1$.

\begin{tabular}{|c|c|c|c|c|}
\hline$\omega L$ & $\frac{\left\langle\left\langle\delta_{u, v} \Phi^{(0,0,0)}\right\rangle\right\rangle}{\left\langle\left\langle\delta_{u, v}\right\rangle\right\rangle}$ & $\frac{\left\langle\left\langle\delta_{u, v} \Phi^{(1,0,0)}\right\rangle\right\rangle}{\left\langle\left\langle\delta_{u, v}\right\rangle\right\rangle}$ & $m_{\mathrm{eff}} L(3.5)$ & $M / \omega$ \\
\hline 6 & $0.1344(5)$ & $0.1343(5)$ & $6.04(5)$ & 0.51 \\
\hline 5 & $0.0168(6)$ & $0.0164(6)$ & $4.2(10)$ & 0.27 \\
\hline
\end{tabular}

Table 4: Simulation results from $10^{7}$ iterations on $24 \times 12^{2}$ lattices.

In the last column we report a mass $M$ which was extracted from the time slice correlation $\left\langle\left\langle\delta_{u_{0}-v_{0}, t}\right\rangle\right\rangle$ without phase factors and using the time periodic $\delta$ function. It also shows a mass-plateau and, as we see, it is smaller than the physical mass. Due to interference effects the spinor correlation decays faster than this 'geometric' one. From this observation one could think that the loops and strings have the 'wrong' size. We tried to generate them with a modified mass parameter $m+\Delta m$ such that $M \approx \omega$ is achieved and then reweighted the observables to the true mass, which is easy if the total monomer number is available. We find however that this simple idea does not improve the sign problem.

\section{Conclusions}

We have formulated the 'worm' algorithm of Prokof'ev and Svistunov for lattice fermions of the Wilson type with Wilson parameter $r=1$. As in the Ising model it estimates stochastically by the Monte Carlo method the untruncated hopping parameter expansion of the partition function together with the graphs needed for the full two-point function, which can thus be computed. The PS algorithm very naturally lends itself to easily keep track of all phase factors and spin matrices that appear in the expansion. In two space time dimensions the contributions of all graphs are positive up to finite size effects and simulations are similarly efficient as in the Ising model. The all order hopping expansion is also worked out and numerically tested in three dimensions. Here the weights of fermion loops acquire complex phase factors and, for small mass and/or large volume lead to numerically uncontrollable fluctuations. The very sharp borderline was found around correlation length two for a $24 \times 12^{2}$ lattice. In particular, the continuum limit cannot be approached. Clearly here the method has to be complemented for instance by an improved estimator which sums some part of the contributions analytically as in cluster methods. No such method is known at present for the system at hand.

For two dimensional fermions we now plan to add the interaction of the GrossNeveu model. For the $\mathrm{O}(N)$ invariant model the Majorana fermion discussed 
here then has to be replicated $N$-fold. For each multi-dimer configuration there are now between $K(x)=0$ and $K(x)=N$ monomers at each site. The four fermion interaction can be enforced by integrating over the common external field $m(x)$ site by site with the appropriate Gaussian weight yielding a $K(x)$-dependent total weight as already discussed in [11]. In this way a coupling between the $N$ 'flavors' arises. The worm head and tail $u$ and $v$ now refer to one of the flavors. When $u=v$ is reached with random re-location also a new flavor-index is chosen randomly. Further details still have to be worked out.

Acknowledgments. I would like to thank Oliver Bär, Rainer Sommer and Willi Rath for discussions. Part of this work was carried out during a one month visit to UCSD (San Diego) and I wish to thank Julius Kuti and the whole high energy physics group for making my stay a most pleasant experience. I would like to thank the Deutsche Forschungsgemeinschaft for support in the framework of SFB Transregio 9.

\section{A Timeslice correlations of free fermions}

We prefer to re-introduce the lattice spacing $a$ for this appendix.

For our numerical test we need to know the free Wilson propagator (13) in the time-momentum basis

$$
\breve{G}\left(x_{0}, p_{k}\right)=a^{D-1} \sum_{x_{k}} G(x) \mathrm{e}^{-i p_{k} x_{k}}
$$

where Latin vector indices $k=1, \ldots, D-1$ refer to spatial components only. Spatial momenta are quantized as demanded by the spatial boundary conditions $\varepsilon_{k}$ and sizes $L_{k}$. From the Fourier expansion of $G$ it is easy to see that $\breve{G}$ is given in terms of a function $f\left(x_{0}\right)$ by

$$
\breve{G}\left(t, p_{k}\right)=\left(-i a \stackrel{\circ}{p}_{k} \gamma_{k}+1+a M\right) f(t)-\frac{1}{2}\left(1+\gamma_{0}\right) f(t+1)-\frac{1}{2}\left(1-\gamma_{0}\right) f(t-1)
$$

with $a \dot{p}_{\mu}=\sin \left(a p_{\mu}\right), M=m+\frac{a}{2} \hat{p}_{k} \hat{p}_{k}, a \hat{p}_{\mu}=2 \sin \left(a p_{\mu} / 2\right)$. The function itself reads

$$
f(t)=\frac{1}{a T} \sum_{p_{0}} \frac{\mathrm{e}^{i p_{0} t}}{\stackrel{\circ}{0}_{0}^{2}+\stackrel{\circ}{p}_{k} \stackrel{\circ}{p}_{k}+\left(M+\frac{a}{2} \hat{p}_{0}^{2}\right)^{2}} .
$$

We find it useful to first evaluate $f$ in the limit of infinite time extent $T=L_{0} \rightarrow \infty$. We demand $1+a M>0$ and introduce

$$
\sinh (a \omega / 2)=\frac{a}{2}\left[\frac{M^{2}+\stackrel{\circ}{p}_{k} \stackrel{\circ}{k}_{k}}{1+a M}\right]^{1 / 2}, \quad a \omega \geqslant 0 .
$$


With $z=\mathrm{e}^{i a p_{0}}$ we now arrive at the contour integral

$$
f_{\infty}(t)=\frac{-1}{1+a M} \oint \frac{d z}{2 \pi i} \frac{z^{t}}{\left(z-\mathrm{e}^{a \omega}\right)\left(z-\mathrm{e}^{-a \omega}\right)}
$$

and obtain

$$
f_{\infty}(t)=\frac{1}{2(1+a M) \sinh a \omega} \mathrm{e}^{-\omega|t|} .
$$

The solution that is (anti)periodic over finite time now follows from

$$
f(t)=\sum_{n=-\infty}^{\infty} f_{\infty}(t+n T)(-1)^{n \varepsilon_{0}}
$$

and reads explicitly for $0 \leqslant t<T$

$$
f(t)=\frac{1}{2(1+a M) \sinh a \omega} \times\left\{\begin{array}{ll}
\cosh [\omega(T / 2-t)] / \sinh (\omega T / 2) & \text { for } \varepsilon_{0}=0 \\
\sinh [\omega(T / 2-t)] / \cosh (\omega T / 2) & \text { for } \varepsilon_{0}=1
\end{array} .\right.
$$

For $0<t<T$ this yields

$$
\operatorname{tr}\left[\breve{G}\left(x_{0}, p_{k}\right)\right]=\frac{M-a \hat{\omega}^{2} / 2}{(1+a M) \dot{\omega}} \times\left\{\begin{array}{lll}
\cosh [\omega(T / 2-t)] / \sinh (\omega T / 2) & \text { for } \varepsilon_{0}=0 \\
\sinh [\omega(T / 2-t)] / \cosh (\omega T / 2) & \text { for } \varepsilon_{0}=1
\end{array}\right.
$$

with $a \hat{\omega}=2 \sinh (a \omega / 2), a \stackrel{\omega}{=}=\sinh (a \omega)$, and

$$
\operatorname{tr}\left[\gamma_{0} \breve{G}\left(x_{0}, p_{k}\right)\right]=\frac{1}{1+a M} \times\left\{\begin{array}{ll}
\sinh [\omega(T / 2-t)] / \sinh (\omega T / 2) & \text { for } \quad \varepsilon_{0}=0 \\
\cosh [\omega(T / 2-t)] / \cosh (\omega T / 2) & \text { for } \varepsilon_{0}=1
\end{array} .\right.
$$

At zero time separation $\operatorname{tr}\left[\gamma_{0} \breve{G}\left(0, p_{k}\right)\right]=0$ vanishes and

$$
\operatorname{tr}\left[\breve{G}\left(0, p_{k}\right)\right]=\frac{1}{1+a M}+\frac{M-a \hat{\omega}^{2} / 2}{(1+a M) \dot{\omega}} \times \begin{cases}\operatorname{coth}[\omega T / 2] & \text { for } \quad \varepsilon_{0}=0 \\ \tanh [\omega T / 2] & \text { for } \quad \varepsilon_{0}=1\end{cases}
$$

Exact results for the zero momentum (for $\varepsilon_{k}=0$ ) correlations

$$
k_{S}\left(x_{0}\right)=-a^{D-1} \sum_{x_{k}}\langle\bar{\xi}(0) \xi(x)\rangle=\operatorname{tr}\left[\breve{G}\left(x_{0}, 0\right)\right]
$$

and

$$
k_{V}\left(x_{0}\right)=-a^{D-1} \sum_{x_{k}}\left\langle\bar{\xi}(0) \gamma_{0} \xi(x)\right\rangle=\operatorname{tr}\left[\gamma_{0} \breve{G}\left(x_{0}, 0\right)\right]
$$

follow from the above formulas.

Incidentally these free field or tree level results form an example how Symanzik improvement for Wilson fermions works. If we multiply the left hand sides of 
(79) and (80) by the wavefunction improvement factor $1+a m$ and eliminate on the right hand side $m$ by the pole mass $m_{\text {pole }}=a^{-1} \ln (1+a m)$, then the finite size continuum limit $\left(T / a \rightarrow \infty\right.$ at fixed $\left.t / T, m_{\text {pole }} T, p_{k} T\right)$ is reached at a rate proportional to $(a / T)^{2}$. For $p_{k}=0$, where $\omega=m_{\text {pole }}$ holds, lattice artifacts are even completely eliminated.

In the correlations above one may also safely take the limit $m \rightarrow 0$ at finite $T$ for antiperiodic boundary conditions $\varepsilon_{0}=1$. This setup may be interpreted as a finite temperature which supplies an infrared regulator, similar as but simpler than in the Schrödinger functional [21. In this limit $k_{V}$ becomes constant while $k_{S}$ vanishes. In $D=2$ the latter may be viewed as a consequence of the discrete chiral symmetry $\xi \rightarrow i \gamma_{0} \gamma_{1} \xi$ under which $k_{S}$ is odd. On the lattice with Wilson fermions this symmetry must emerge in the continuum limit at the critical mass, zero in the free case. Due to the (accidental) complete improvement it here holds already on the lattice at all nonzero separations.

As discussed in [16] $k_{S}, k_{V}$ may be used to formulate massless nonperturbative renormalization conditions in the interacting Gross-Neveu model. The field can be normalized by keeping for instance $k_{V}(T / 2)=1$ while the critical mass may be determined by tuning to $k_{S}(T / 4)=0$. Note that $k_{S}(T / 2)$ vanishes due to time reflection invariance combined with antiperiodicity for all mass values and is not suitable to fix the chiral point.

\section{B Spin phase: continuum and lattice}

We now consider an alternative way to compute the loop phase (23)

$$
w(\lambda)=-\operatorname{tr}\left[P\left(m_{1}\right) P\left(m_{2}\right) \cdots P\left(m_{|\lambda|}\right)\right]
$$

from the sequence of lattice unit vectors $m_{i}$. An earlier discussion of this quantity is found in [22]. As a technical simplification we assume for a while that straight sections are absent, $m_{i+1} \cdot m_{i}=0$. We can rotate one projector into the next, for example

$$
P\left(m_{1}\right) P\left(m_{2}\right)=R\left(m_{1}, m_{2}\right) P\left(m_{2}\right) R\left(m_{1}, m_{2}\right)^{-1} P\left(m_{2}\right)=\frac{1}{\sqrt{2}} R\left(m_{1}, m_{2}\right) P\left(m_{2}\right)
$$

with the $\pi / 2$ spinor rotation matrix

$$
R\left(m_{1}, m_{2}\right)=\mathrm{e}^{\frac{\pi}{8}\left[\not m_{1}, \not h_{2}\right]}
$$

in the usual notation $\not h=m_{\mu} \gamma_{\mu}$. By iterating this step we arrive at

$$
-w(\lambda)=2^{-(\mid \lambda \vdash 1) / 2} \operatorname{tr}\left[R_{\mathrm{loop}} R\left(m_{1}, m_{|\lambda|}\right) P\left(m_{|\lambda|}\right)\right]
$$


with

$$
R_{\text {loop }}=R\left(m_{1}, m_{2}\right) R\left(m_{2}, m_{3}\right) \cdots R\left(m_{|\lambda|-1}, m_{|\lambda|}\right) R\left(m_{|\lambda|}, m_{1}\right) .
$$

As the loop closes this total rotation must bring $m_{1}$ back to itself, it belongs to the stability group of this direction.

The rotations introduced in (86) can be characterized by mapping a cube onto itself by rotations by $\pi / 2$ through any lattice plane. Products of such operations do not preserve the form (86) but are general members of the cubic group. The number of elements is given by twice the number of orientations of a $D$-dimensional cube along the lattice axes (without reflections) that is $2^{D} D$ ! in $D$ dimensions. The twofold multiplicity is because $\pm R$ in the spinor representation corresponds to only one re-orientation of the cube as usual with spinor rotations.

In $D=2$ the cubic group is abelian and has 8 elements. Here $R_{\text {loop }}$ adds up the half-angles of the rotations that a test-particle taken around the loop experiences. For a non-intersecting closed loop we get $R_{\text {loop }}=-1$ for a $2 \pi$ rotation. Then we find in total

$$
w(\lambda)=(-)^{\nu+1} 2^{-C(\lambda) / 2}
$$

with the number of complete rotations $\nu$ and the number of corners along the loop $C(\lambda)$. This form of the result now also covers the case where $m_{i+1}=m_{i}$ can occur, a straight section not counted in $C(\lambda)$.

In $D=3$ the cubic group is non-abelian and has 48 elements. Now $R_{\text {loop }}$ can only be a rotation around the $m_{1}$ axis,

$$
R_{\text {loop }}= \pm \mathrm{e}^{i \frac{\alpha}{2} \not h_{1}}
$$

with $\alpha$ a multiple of $\pi / 2$. Then one easily evaluates

$$
\operatorname{tr}\left[R_{\text {loop }} R\left(m_{1}, m_{|\lambda|}\right) P\left(m_{|\lambda|}\right)\right]=\frac{1}{\sqrt{2}} \mathrm{e}^{-i \frac{\alpha}{2}}
$$

The sign ambiguity of the spinor transformation ( 2 to 1 covering) has been absorbed into the $\alpha$ and the phase is in Z(8). For $m_{1}=m_{|\lambda|}$ again the factor $1 / \sqrt{2}$ has to be dropped.

It is interesting to note that a strictly analogous construction can be made in the continuum. Let us assume a closed curve $\vec{\gamma}(t), t \in[0,1]$. For a regular parametrization we have $\dot{\vec{\gamma}} \neq 0$ and form $m(t)=\dot{\vec{\gamma}} /|\dot{\vec{\gamma}}|$, a mapping from $S_{1}$ to $S_{D-1}$. We now move $m(t)$ along the loop by infinitesimal rotations in spinor form

$$
\dot{\not h}=\dot{\not h} \not \varkappa^{2}=\frac{1}{2}[\dot{\not h} \not h, \not h]
$$

using $\dot{m} \cdot m=0$. The solution of

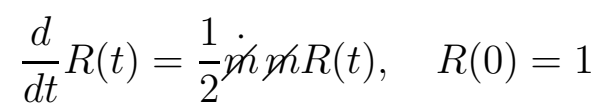


is formally given by the path-ordered integral

$$
R(t)=\mathcal{P} \exp \left\{\frac{1}{2} \int_{0}^{t} d \tau \dot{\not h}(\tau) \not h h(\tau)\right\} .
$$

The direction $m$ is parallel transported according to

$$
\not h(t)=R(t) \not h(0) R^{-1}(t)
$$

At $t=1$ we must recover $\not h(1)=\not h(0)$ and thus $R(1)$ must induce a rotation that has $m(0)$ as a fixed point. In $D=2$ there is no nontrivial such rotation, but since both $R=1$ and $R=-1$ induce the trivial rotation of $m(0)$, it is possible to find $R(1)=-1$. Indeed the latter result emerges for a loop with an odd number of windings. In $D=3 R(1)$ can induce an arbitrary rotation around the $m(0)$ axis, $R(1)=\exp [i \alpha \not h(0) / 2]$. Thus there is a phase, now a general element of $\mathrm{U}(1)$, associated with a closed loop. It does not depend on where the loop is cut by the parametrization: going to another point, $R(1)$ just gets similarity-transformed. It is a property just of the loop itself. It seems very likely that this is a known concept in differential geometry, however no reference is known to the author.

\section{References}

[1] S. A. Gottlieb, W. Liu, D. Toussaint, R. L. Renken, R. L. Sugar, Hybrid Molecular Dynamics Algorithms for the Numerical Simulation of Quantum Chromodynamics, Phys. Rev. D35 (1987) 2531-2542.

[2] S. Duane, A. D. Kennedy, B. J. Pendleton, D. Roweth, Hybrid Monte Carlo, Phys. Lett. B195 (1987) 216-222.

[3] J. Hirsch, R. Sugar, D. Scalapino, R. Blankenbecler, Monte Carlo Simulations of one-dimensional Fermion Systems, Phys. Rev. B 26 (9) (1982) 5033-5055.

[4] P. Rossi, U. Wolff, Lattice QCD with Fermions at Strong Coupling: A Dimer System, Nucl. Phys. B248 (1984) 105.

[5] H. G. Evertz, The Loop Algorithm, Adv. Phys. 52 (2003) 1.

[6] D. H. Adams, S. Chandrasekharan, Chiral Limit of strongly coupled Lattice Gauge Theories, Nucl. Phys. B662 (2003) 220-246.

[7] M. Karowski, R. Schrader, H. J. Thun, Monte Carlo Simulations for Quantum Field Theories involving Fermions, Commun. Math. Phys. 97 (1985) 5. 
[8] C. Gattringer, Loop Representation for 2-d Wilson Lattice Fermions in a scalar Background Field, Nucl. Phys. B543 (1999) 533-542.

[9] K. Scharnhorst, The exact Equivalence of the one-flavour Lattice Thirring Model with Wilson Fermions to a two-colour Loop Model, Nucl. Phys. B503 (1997) 479-504.

[10] C. Gattringer, V. Hermann, M. Limmer, Fermion Loop Simulation of the Lattice Gross-Neveu model, Phys. Rev. D76 (2007) 014503.

[11] U. Wolff, Cluster Simulation of Relativistic Fermions in Two Space-Time Dimensions, Nucl. Phys. B789 (2008) 258-276.

[12] W. Rath, Diploma thesis, in preparation, HU Berlin, 2009.

[13] N. Prokof'ev, B. Svistunov, Worm Algorithms for Classical Statistical Models, Phys. Rev. Lett. 87 (16) (2001) 160601.

[14] U. Wolff, Simulating the All-order strong Coupling Expansion I: Ising Model Demo, Nucl. Phys. B In Press (2008).

[15] S. Chandrasekharan, U.-J. Wiese, Meron-cluster Solution of a Fermion Sign Problem, Phys. Rev. Lett. 83 (1999) 3116-3119.

[16] T. Korzec, U. Wolff, Gross-Neveu Model as a Laboratory for Fermion Discretization, PoS LAT2006 (2006) 218.

[17] T. Korzec, Precise Determination of universal finite Volume Observables in the Gross-Neveu Model, PhD Thesis, 2007, http://nbn-resolving.de/urn:nbn:de:kobv:11-10078995.

[18] S. Samuel, The Use of anticommuting Integrals in statistical Mechanics. 1, J. Math. Phys. 21 (1980) 2806-2814.

[19] Y. Deng, T. M. Garoni, A. D. Sokal, Dynamic Critical Behavior of the Worm Algorithm for the Ising Model, Phys. Rev. Lett. 99 (2007) 110601.

[20] U. Wolff, Monte Carlo Errors with less Errors, Comput. Phys. Commun. 156 (2004) 143-153.

[21] M. Lüscher, R. Narayanan, P. Weisz, U. Wolff, The Schrödinger functional: A renormalizable Probe for non-Abelian Gauge Theories, Nucl. Phys. B384 (1992) 168-228.

[22] I. O. Stamatescu, A Note on the Lattice fermionic Determinant, Phys. Rev. D25 (1982) 1130. 